\title{
How does the viability of bone-restricted osteocytes regulate bone atrophy?
}

Prof. Dr Aline Bozec and Dr Darja Andreev

Universitätsklinikum Erlangen and Friedrich-Alexander

University Erlangen-Nürnberg

Although the skeleton appears stiff and solid, it is, in fact, a dynamic and sensitive network,

demanding a fine balance between the bone-resorbing osteoclasts and the bone-forming osteoblasts. Disruption of this homeostasis frequently leads to exaggerated bone degradation by osteoclasts,

manifesting in pathological bone loss. Bone metabolism is largely controlled by osteocytes that are terminally differentiated osteoblasts, becoming trapped within the bone matrix (Figure 1).

Osteocytes represent the major cell type within the bone compartment. Nonetheless, for a long time, they were neglected from the field of bone research esed from the field of bone research because they appear biologically inert. Today, their broad contribution to bone remodelling is widely recognised. In particular, osteocytes act as mechanosensors of bone, participate regulate osteoblasts and especially osteoclasts (Bonewald, 2011).

The rather inactive feature of osteocyte allows them to live an average half-life of 25 years. Despite their longevity, excessive osteocyte death is observed upon ageing, notably in bone diseases loss, such as osteoporosis, arthritis, fracture, and osteonecrosis (Figure 2A). Up to now, it is not yet known whether increased osteocyte death appears just as a concomitant of bone disease or may even contribute to the disease pathology.

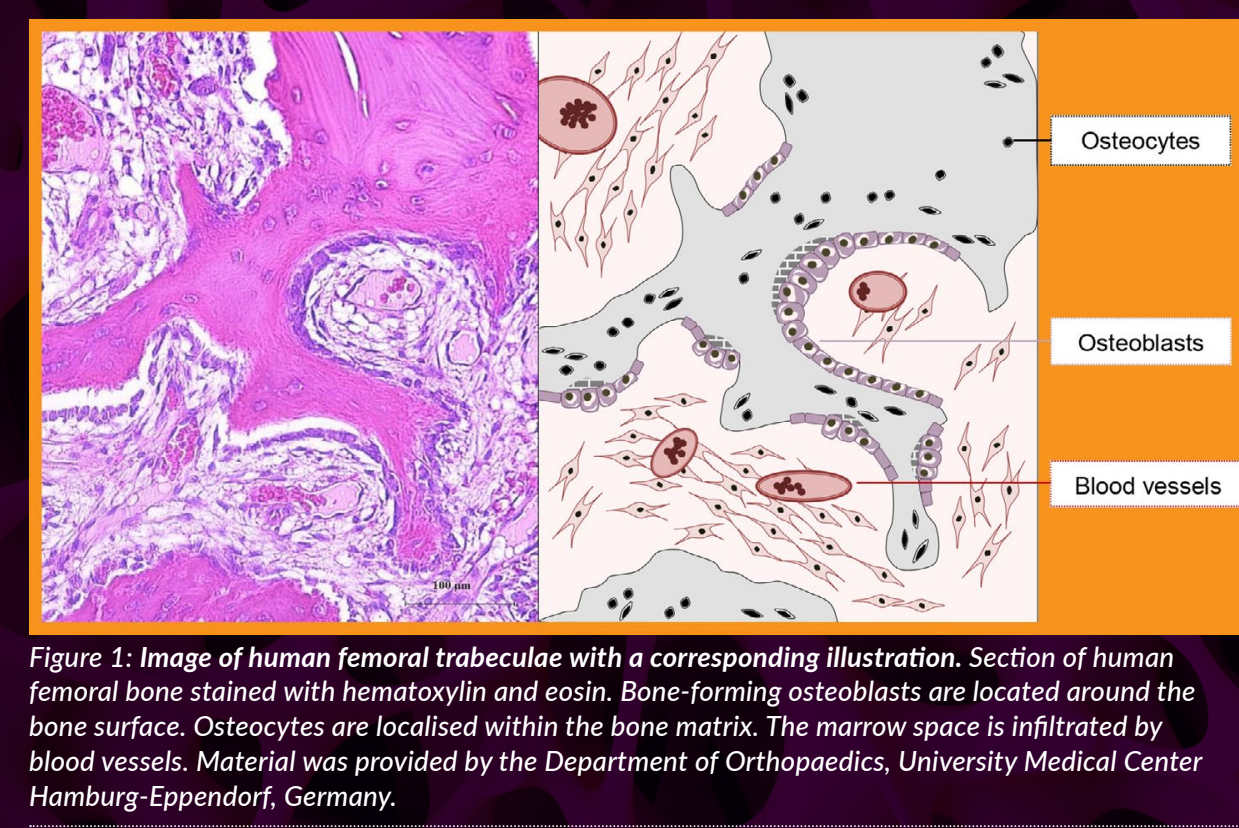

\section{The ODE project}

For this purpose, we initiated the ERC Starting Grant project "Unknov delinot in which maner ostecytes ide with the bone matrix and which conseques their death has for bone health.
So far, the death of osteocytes is still a mystery. It is believed that factors like oxygen level and nutrients distributed by blood vessels within the bone strongly influence their viability. When shortages occur, osteortes undero auto to protecy damage. Hower, when the from is too severe, the death of the cells is initiated (Komori, 2016). One of the primary goals of the ODE project is delineate in detall the death process osteocytes in dependence of different bone conditions, such as arthritis, fracture and osteonecrosis. The use of newly developed imaging techniques and omics platforms will allow us to analyse osteocytes within their remote localisation in the bone matrix. With this study, we want to answer question ke. Which stress factors (hypoxia, osteocyte death? Which death pathway apoptosis, necroptosis, ferroptosis, necrosis) is specifically triggered in osteocytes? Is osteocyte viability important for healthy bone composition and how is it affected by bone disease?

Generally, the body gets rid of dead cells very quickly by specialised phagocytic cells to prevent sterile inflammation However, bone is exceptional since phagocytes cannot reach the isolated osteocytes within the mineralised tissue. Thus, at some point, osteocytes are supposed to burst, releasing damageassociated molecular patterns (DAMPs) into the surrounding, thereby priming an inflammatory environment. Last year we published an article describing for the first time that osteocytic DAMPs are able to stimulate osteoclast formation (Andreev et al., 2020). This response is mediated by et al., 2020). This response is mediated by the C-type lectin receptor, macrophageinducible C-type lectin (short Mincle, expressed on osteoclasts (Figure 2B). As a pattern recognition receptor (PRR) Mincle is able to recognise microbi compounds as well as DAMPs. These results opened many new questions that will be addressed within the ODE project as: Which DAMPs are released by osteocytes upon death? Are other DAMP sensing PRRs besides Mincle expressed

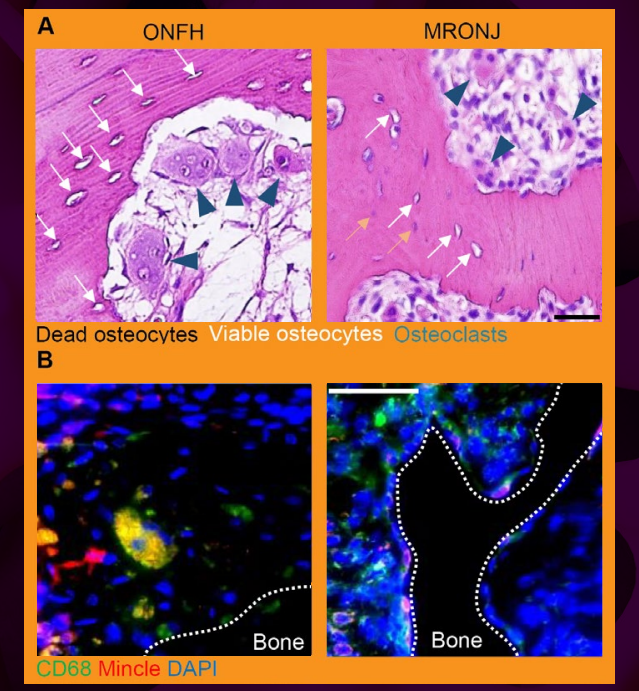

PROJECT NAME

cyte DEath (ODE)

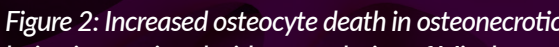
Pson is assocthed we hipregulation of Mincle-
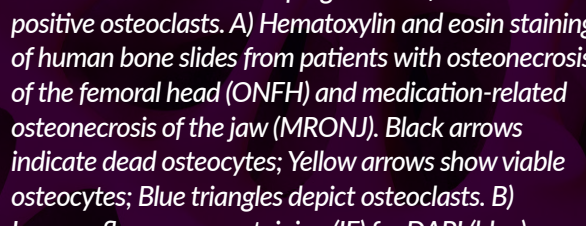

Immanofluoressence staining (IF) for DAPI (blue,

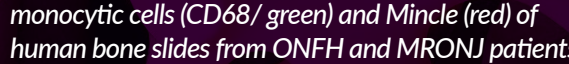

apted from Andreev et al., 2020.

on osteoclasts and have an impact on osteoclast biology?

Conclusively, the ODE project will provide a new understanding of the sensitive balance of bone homeostasis and how stress factors in bone such as inflammation and reduced vascularisation influence the viability of osteocytes. We will decipher in detail which impact the death of osteocytes has on the bone environment and bone strength and how we can avod this negative infuence. On a broader scale, we can use the knowledge gained to better understand the pathogents of bone loss and otential new pathways that ted in the future to prevent bone loss from happening.

\section{References}

Andreev, D., Liu, M., Weidner, D., Kachler, K., Faas, M., Gruneboom, A., et al. (2020) 'Osteocyte Necrosis
Triggers Osteoclast-mediated Bone Loss Through Macrophage-Inducible C-type Lectin', The Journal of Clinical Investigation. 130(9), pp.4811-30.

Bonewald, L.F. (2011) 'The Amazing Osteocyte', Journal of Bone and Mineral Research: The Offficial Journal Komori, T. (2016) 'Cell Death in Chondrocytes, Osteoblasts, and Osteocytes', International Journal of

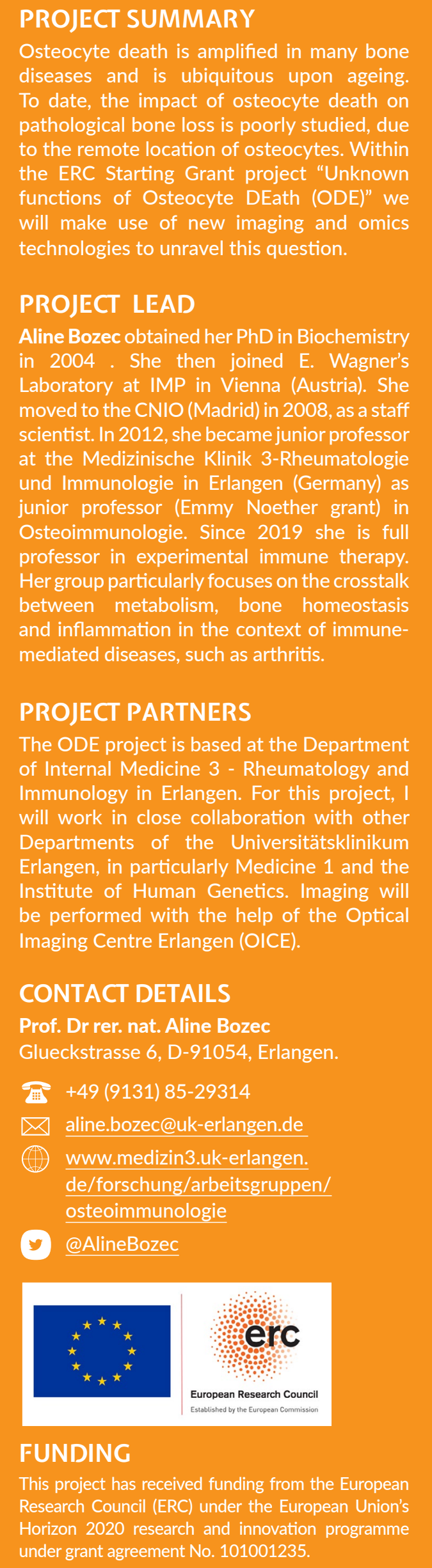

